Original Paper http://ajol.info/index.php/ijbcs http://indexmedicus.afro.who.int

\title{
Effet de la supplémentation des rations par un additif alimentaire sur la croissance pondérale et le rendement carcasse des chevreaux Roux de Maradi au Niger
}

\author{
Aminatou Aliou BARAZI ${ }^{1^{*}}$, Mahamadou CHAIBOU ${ }^{2}$, \\ Ousseini Mahaman M. MOUCTARI ${ }^{2}$ et Moussa Elhadji NOUHOU ${ }^{3}$ \\ ${ }^{I}$ Ministere de l'Agriculture et de l'Elevage/Direction Générale de Production et des Industrie \\ Animales/Programme National d'Amélioration Génétique /Bovins Locaux, BP : 12091 \\ ${ }^{2}$ Département Productions Animales, Faculté d'Agronomie, Université Abdou Moumouni, \\ BP : 10960, Niamey, Niger. \\ ${ }^{3}$ Centre Secondaire d'Elevage Caprin de Maradi, Niger. \\ *Auteur correspondant ; E-mail: baraminaly@yahoo.fr
}

\section{RESUME}

Au Niger, la longue saison sèche rend difficile l'alimentation des animaux : les pâturages, base de la ration alimentaire sont trop pauvres pour couvrir les besoins de production qualitatifs et quantitatifs des animaux. Cette étude porte sur l'incorporation de différentes doses d'acide malique dans la ration alimentaire des chevreaux roux de Maradi afin d'évaluer son effet sur l'ingestion des rations, l'indice de consommation, la croissance et le rendement carcasse. Quarante (40) chevreaux d'âge compris entre 7 à 8 mois et d'un poids vif moyen de $10,77 \pm 0,19 \mathrm{~kg}$ ont été répartis au hasard en quatre (4) lots de 10 têtes chacun. Les rations distribuées sont composées de fane de niébé, de son de blé et de l'acide malique. La ration du lot témoin (T0) ne contenait pas d'acide malique. Les rations des lots T1, T2 et T3 comportent respectivement 3, 6 et $9 \mathrm{~g}$ d'acides malique par $\mathrm{kg}$ de concentré. A l'issue de l'expérimentation qui a duré 240 jours, les animaux soumis au traitement T3 ont obtenu le meilleur gain moyen quotidien (GMQ) soit 117,42 \pm 31,04 g/jr suivi des animaux du lot T2 $(109,57 \pm 26,98 \mathrm{~g} / \mathrm{jr})$, puis les animaux du lot témoin T0 $(102,85 \pm 25,01 \mathrm{~g} / \mathrm{jr})$ et ceux du lot $\mathrm{T} 1(86,21 \pm 19,47 \mathrm{~g} / \mathrm{jr})$. Les analyses n'ont montré aucune différence significative au seuil de $5 \%$ entre les lots supplémentés et le témoin pour les paramètres comme la consommation alimentaire $(\mathrm{P}=0,951)$, le poids corporel $(\mathrm{P}=1,000)$ et le poids des carcasses $(\mathrm{P}=0,68)$. L'indice de consommation de fane et son de blé le plus élevé a été obtenu avec le lot témoin respectivement 9,12 et 6,06. L'acide malique peut être incorporé à une dose allant jusqu'à $9 \mathrm{~g} / \mathrm{kg}$ de ration et permettre ainsi l'assimilation des aliments et l'obtention d'une bonne performance de croissance.

(C) 2019 International Formulae Group. All rights reserved

Mots clés : Chevreaux roux, ration, acide malique, supplémentation, Maradi, Niger.

\section{Supplementation by a food additive on weight gain and carcass yield of redhead kids in Maradi, Niger}

\begin{abstract}
In Niger, the long dry season makes it difficult to feed animals: pastures, the basis of the food ration, are too poor to cover the qualitative and quantitative production needs of animals. This study investigates the
\end{abstract}


incorporation of different doses of malic acid into the diet of redhead kids in Maradi to evaluate its effect on dietary intake, consumption index, growth and carcass yield. Forty (40) kids between 7 and 8 months old with an average live weight of $10.77 \pm 0.19 \mathrm{~kg}$ were randomly assigned to four (4) batches of 10 heads each. The feeds distributed are composed of bean top, wheat bran and malic acid. The feed of the control batch (T0) did not contain malic acid. The feeds of batches T1, T2 and T3 comprise respectively 3, 6 and $9 \mathrm{~g}$ of malic acid per $\mathrm{kg}$ of concentrate. At the end of the experiment, which lasted 240 days, the animals subjected to the T3 treatment obtained the best average daily gain (GMQ) ie $117.42 \pm 31.04 \mathrm{~g} /$ day followed by the animals of the T2 batch $(109,57 \pm 26.98 \mathrm{~g} /$ day $)$, then the animals of control batch T0 (102.85 $\pm 25.01 \mathrm{~g} /$ day $)$ and those of batch T1 $(86.21 \pm 19.47 \mathrm{~g} /$ day). No significant difference is showed by the analysis at the $5 \%$ level between the supplemented batches and the control batch for parameters such as food consumption $(\mathrm{P}=0.951)$, body weight $(\mathrm{P}=1.000)$ and carcass weight $(\mathrm{P}=0.68)$. The highest consumption index of bean top and wheat bran was obtained with the control group respectively 9, 12 and 6, 06. Malic acid can be incorporated at a dose of up to $9 \mathrm{~g} / \mathrm{kg}$ of diet and thus allow the assimilation of food and obtaining a good growth performance.

(C) 2019 International Formulae Group. All rights reserved

Keywords: Redhead kids, feed, malic acid, supplementation, Maradi, Niger.

\section{INTRODUCTION}

L'alimentation constitue l'un des principaux facteurs de réussite dans une activité d'élevage. En effet, même quand l'éleveur dispose de races à hautes performances de production et que les animaux sont en bonne santé, il ne peut obtenir de rendements maximum en lait ou viande que si ses animaux sont bien nourris. Dans les pays sahéliens comme le Niger, la longue saison sèche rend difficile l'alimentation des animaux : les pâturages qui sont la base de la ration alimentaire sont trop pauvres et moins riches pour couvrir les besoins de production (qualitatifs et quantitatifs) des animaux (CIRDES, 2005). Chez les ruminants, la principale limite à l'expression optimale du potentiel génétique est l'apport insuffisant de nutriments. La couverture des besoins nutritionnels des animaux les plus performants passe par l'utilisation d'aliments concentrés et par une augmentation du niveau d'ingestion. Cependant, cette situation peut conduire à l'apparition de troubles digestifs d'où la nécessité d'emploi d'additifs destinés à réguler le fonctionnement de l'écosystème microbien ruminal et à stimuler l'ingestion des aliments par les animaux. Ainsi l'utilisation de suppléments alimentaires alternatifs composés d'enzymes et d'acides organiques est en augmentation dans de nombreux pays (AFSSA, 2007). Les acides organiques ont des effets antibactériens sur le système digestif des animaux. En plus de leurs effets stimulateurs de croissance, ils peuvent également être utilisés comme sources de minéraux, d'énergie et même comme agents conservateurs (Papatsiros et Christodoulopoulos, 2011). Certains acides organiques comme l'acide malique, l'acide fumique et l'acide aspartique sont généralement présents dans la nature en tant que constituants normaux des tissus végétaux et animaux (Aksu et al., 2012). Le potentiel des acides organiques notamment l'acide fumarique ou l'acide malique en tant qu'inhibiteurs de la méthanogènese in vitro (Carro et Ranilla, 2003 ; Newbold et al., 2005) et in vivo (Lila et al., 2004 ; Wallace et al., 2006) a été bien signalé. Il est indiqué que les acides organiques et leurs sels sont perçus comme une alternative aux facteurs de croissance antibiotiques en nutrition animale (Indresh, 2007). Des travaux antérieurs avaient déjà rapporté que les acides citriques et malique étaient les principaux acide organiques (Houngbo et al., 2018). Si des études sur l'utilisation de l'acide malique et de ses sels comme produits antibactériens ou comme source minérale dans l'alimentation animale ont été effectuées (Aksu et al., 2012), il n'en demeure pas moins important qu'il peut être source d'énergie ou facteur de croissance lorsqu'il est incorporé dans des rations alimentaires. C'est ainsi qu'une l'étude expérimentale a été conduite au Centre Secondaire d'Elevage Caprin (CSEC) de Maradi afin de tester l'effet d'acide malique 
sur la croissance pondérale et le rendement carcasse des chevreaux Roux.

\section{MATERIEL ET METHODES \\ Matériel \\ Site expérimental}

L'étude a été conduite au Centre Secondaire d'Elevage Caprin (CSEC) de Maradi situé à 3,5 km à l'Est de la ville de Maradi et couvre une superficie totale de 1850 ha dont 150 ha affectés à la station avicole (Moussa, 2011). Le centre est divisé en quatre (4) points d'élevage appelés chèvreries, dont trois (3) opérationnelles (Plan CSEC). Ainsi, les chèvreries sont reparties en 4 points : $\mathrm{X}, \mathrm{Y}$, $\mathrm{Z}, \mathrm{W}$. Dix (10) chèvreries de $100 \mathrm{~m}^{2}$ chacune existent au point d'élevage $X$. Le point d'élevage Y comprend douze (12) chèvreries de $50 \mathrm{~m}^{2}$ chacune et le point d'élevage $\mathrm{Z}$ dispose de huit (8) chèvreries de $50 \mathrm{~m}^{2}$ chacune. Le point d'élevage $\mathrm{W}$, inachevé, n'est pas encore opérationnel (Marc, 2001). La région de Maradi est située au centre sud du Niger et à environ $600 \mathrm{~km}$ à l'Est de Niamey, entre les parallèles $13^{\circ}$ et $15^{\circ} 26^{\prime}$ de latitude Nord et les méridiens $6^{\circ} 16^{\prime}$ et $8^{\circ} 36^{\prime}$ de longitude Est (Ali et al., 2003; Nouhou, 2014 ; Abdou, 2014). Elle est limitée à l'Est par la région de Zinder, à l'Ouest par la région de Tahoua, au Nord par la région d'Agadez et au Sud par la République Fédérale du Nigeria sur environ $150 \mathrm{Km}$ de frontière poreuse. (OCHA, 2014). Le climat est caractérisé par une saison de pluie qui dure environ 4 mois (fin mai-début octobre) avec une moyenne annuelle de $620 \mathrm{~mm}$. Les températures extrêmes varient entre 25 et $45^{\circ} \mathrm{C}$ (Mainet, 1967).

\section{Animaux}

Quarante (40) chevreaux roux âgés de 7 mois en moyenne avec un poids vif moyen de $10,77 \pm 0,19 \mathrm{~kg}$ ont été utilisés. Ces chevreaux portent des boucles numérotées avec des couleurs différentes pour leur identification inter lots. Chaque chevreau était logé dans une cage métabolique individuelle grillagée mesurant chacune $2,20 \mathrm{~m} \mathrm{x} \mathrm{1,60} \mathrm{m.}$ Ces animaux ont été déparasités avec de l'albendazole $300 \mathrm{mg}$.

\section{Aliments}

Les aliments utilisés pour la conduite de cette expérimentation sont composés de fane de niébé comme aliment de base et du son de blé comme concentré.

Le son de blé a été acheté auprès des commerçants sur le marché local de Maradi. Les fanes de niébé ont été achetées auprès des agriculteurs des villages environnant du Centre. Ces fanes, ont été préalablement broyées de manière sommaire avant d'être distribuées. Ceci pour rendre homogène la présentation physique de ces fanes par rapport au son de blé afin d'éviter un effet négatif sur la consommation alimentaire liée à la présentation physique de l'aliment.

\section{Autre matériel}

- Des mangeoires individuelles en matière plastique et métalliques ont été utilisées respectivement pour la distribution du son et des fanes;

- Des abreuvoirs d'une capacité de 15 litres chacun placés dans chaque compartiment pour abreuver à volonté les animaux ;

- Des balances pour la pesée des aliments, les refus et aussi une balance sensible pour peser l'additif alimentaire.

\section{Méthode \\ Dispositif expérimental et collecte des données}

L'essai a été conduit selon un dispositif expérimental des blocs complets randomisés. Les animaux ont été répartis de façon aléatoire en quatre (4) lots de 10 chacun et en deux rangées dans des cages métaboliques individuelles grillagées. Les animaux ont été pesés en début de l'essai après une semaine d'adaptation. Au cours de l'expérimentation les animaux ont été pesés à jeûne, chaque deux semaines pour suivre la croissance pondérale. Quatre rations ont été formulées. $\mathrm{La}$ ration témoin $\mathrm{T} 0$ ne contenait que des fanes de niébé et du son de blé tandis que les rations $\mathrm{T} 1, \mathrm{~T} 2$ et $\mathrm{T} 3$ contenaient en plus des fanes et du son de blé des doses d'acide malique respectivement de 3,6 et $9 \mathrm{~g} / \mathrm{kg}$ de matière sèche incorporés dans le son (Tableau $1)$.

Chaque ration était offerte une fois en 24 heures. Les fanes ont été apportées les matins à partir de 7 heures et les concentrés dans l'après-midi à partir de $14 \mathrm{~h} 30 \mathrm{mn}$. Chaque aliment et ses refus ainsi que les fèces 
de chaque animal ont été collectés et pesées quotidiennement.

\section{Analyses bromatologiques}

Les analyses de la composition chimique des aliments ont concerné les fanes de niébé et le son de blé utilisés dans l'essai. Ces analyses effectuées au Laboratoire d'Alimentation Animale de la Faculté d'Agronomie de l'Université Abdou Moumouni de Niamey ont porté sur la matière sèche (MS), la matière minérale (MM), la cellulose brute $(\mathrm{CB})$ et la matière grasse $(\mathrm{MG})$. La teneur en matière sèche (MS) a été déterminée en séchant $5 \mathrm{~g}$ d'échantillon à l'étuve pendant trois heures à $103{ }^{\circ} \mathrm{C}$. Les cendres ont été obtenues par incinération de 3 $\mathrm{g}$ de l'échantillon au four à $550{ }^{\circ} \mathrm{C}$ pendant 4 heures. La teneur en matière organique est obtenue à partir des résultats de la matière minérale ou cendre (AOAC, 1990). La cellulose brute $(\mathrm{CB})$ a été déterminée selon la méthode de Weende (SEWA, 1860) et les protéines brutes (PB), calculées en multipliant par 6,25 la teneur en azote total obtenue par la méthode de Kjeldahl (AOAC, 1990). La détermination de la teneur en matière grasse est effectuée selon la méthode d'extraction par le soxhlet en utilisant l'hexane comme solvant à reflux (AOAC, 1990).

\section{Evaluation des paramètres zootechniques}

Les données collectées ont permis de calculer les quantités d'aliment consommées (CA), les Gains Moyens Quotidiens (GMQ), les rendements carcasses moyens (RC), et l'indice de consommation (IC).

Consommation alimentaire individuelle (CA)

La détermination de la consommation alimentaire ou quantité d'aliments ingérés a été obtenue en faisant la différence entre les quantités d'aliments distribués (QAd) quotidiennement et le refus (QAr).

$$
\mathrm{CA}(\mathrm{kg})=\mathrm{QAd}-\mathrm{QAr}
$$

\section{Indice de consommation (IC)}

Il a été calculé en faisant le rapport de la quantité moyenne d'aliment consommée pendant une période sur le gain de poids moyen durant la même période.

$$
\text { IC }=\frac{\text { QACP }(\mathrm{g})}{\text { GPP }(\mathrm{g})}
$$

avec QACP : Quantité d'aliment consommée pendant une période ;

GPP : Gain de poids durant la même période.

\section{Gain Moyen Quotidien (GMQ)}

Les animaux ont été pesés à jeûne, au démarrage et à la fin de chaque deux semaines, avant la distribution de la ration du jour à $7 \mathrm{~h}$ $30 \mathrm{mn}$. Le poids de chaque animal est alors enregistré afin de suivre sa croissance pondérale. Cette dernière a permis de déterminer le gain moyen quotidien (GMQ). Ce gain de poids moyen quotidien ou gain de croît journalier donne une indication sur la vitesse de croissance d'un animal sur une période donnée. Il est défini comme le différentiel de croît entre deux dates (Chaibou, 2015). Le calcul de GMQ entre la période 1 (âge 1) et la période 2 (âge 2) a été fait selon la formule suivante :

$$
\begin{aligned}
& \text { Poids } 2 \text { - Poids } 1 \\
& \text { GMQ (g/jour) = -------- Age } 1
\end{aligned}
$$

\section{Rendement carcasse}

Pour étudier le rendement carcasse, les animaux sont abattus, habillés et éviscérés afin d'obtenir la carcasse avec ses 4 quartiers. Ainsi après abattage, le rendement (\%) carcasse pour chaque lot a été déterminé. Un échantillon de 50\% des animaux du test c'està-dire 5 par lot a été constitué. Les animaux de cet échantillon ont été abattus pour étudier le rendement. Ce résultat a été calculé en faisant le rapport du poids carcasse obtenu après éviscération et le poids vif de l'animal à l'abattage soit :

$$
\begin{aligned}
& \text { PCE }(\mathrm{g}) \\
& \mathrm{RC}(\%)=-\frac{\mathrm{PVAA}(\mathrm{g})}{\mathrm{-a}} 100 \\
& \text { avec PCE : Poids de la carcasse après } \\
& \text { éviscération; } \\
& \text { PVAA : Poids vif de l'animal à l'abattage. }
\end{aligned}
$$

\section{Analyse statistique}

Les données ont été saisies sur le tableur «EXCEL». Le calcul des moyennes, des écarts types, l'analyse de variances et la comparaison des moyennes (Test de Duncan à travers le model linéaire général) ont été réalisés à l'aide du logiciel Gen Stat. Les moyennes sont comparées au seuil de 5\%. 
Tableau 1 : Composition des différentes rations.

\begin{tabular}{llll}
\hline Traitements & Fane de niébé $(\%)$ & son de blé $(\%)$ & $\begin{array}{l}\text { Acide }(\mathbf{g} / \mathbf{k g} \text { de matière } \\
\text { sèche de concentré })\end{array}$ \\
\hline T0 & 60 & 40 & 0 \\
\hline T1 & 60 & 40 & 3 \\
\hline T2 & 60 & 40 & 6 \\
\hline T3 & 60 & 40 & 9 \\
\hline
\end{tabular}

\section{RESULTATS}

\section{Composition chimique du son de blé et des fanes de niébé}

Les résultats issus des analyses bromatologiques sont indiqués dans le Tableau 2. La composition chimique des aliments utilisés au cours de l'essai indiqués dans le tableau 2 montre que la teneur en matière sèche des fanes de niébé est supérieure à celle du son de blé. On constate également que les fanes sont plus riches en celluloses brute $(35,14 \%)$ que le son de blé $(15,42 \%)$. Cependant concernant la matière azotée totale, le son présente des teneurs plus importantes que les fanes de niébé respectivement 18,71 et $17,21 \%$.

\section{Effets de l'additif sur les paramètres évalués \\ Consommation alimentaire et Indice de consommation}

Les moyennes de l'ingestion d'aliment en gramme pour l'ensemble des lots rapportées dans le Tableau 3 montrent que, la ration dans laquelle les fanes sont plus ingérées est celle du lot témoin T0 (417,30 g) et la consommation élevée du son de blé a été constatée au niveau du T3 ayant reçu $9 \mathrm{~g}$ d'acide malique par $\mathrm{kg}$ de concentré en matière sèche. L'analyse statistique n'a montré aucune différence significative entre les lots. Les indices de consommation les plus élevés pour les fanes et le son de blé sont obtenus avec les animaux du lot témoin soit respectivement 9,12 et 6,06.

\section{Evolution pondérale des animaux}

Les poids moyens des animaux par lot à la fin de l'expérimentation sont de 19,96 \pm $2,92 \mathrm{~kg}, 19,78 \pm 3,10 \mathrm{~kg}, 20,71 \pm 3,20 \mathrm{~kg}$ et $20,40 \pm 2,96 \mathrm{~kg}$, respectivement pour le lot témoin du T0 et les animaux des lots T1, T2, et T3 ayant reçus l'additif. Ces animaux avaient en début de l'essai les poids moyens respectifs de $10,88 \pm 2,30 \mathrm{~kg} ; 10,50 \pm 1,27 \mathrm{~kg}$; $10,92 \pm 1,86 \mathrm{~kg}$ et $10,78 \pm 1,81 \mathrm{~kg}$. L'analyse statistique montre qu'il n'y a pas de différence significative au seul de 5\% entre les lots, $(\mathrm{P}=$ $1,000)$.

L'analyse de la Figure 1 montre que le poids moyen des animaux des lots $\mathrm{T} 1$ et $\mathrm{T} 2$ ont connus une légère diminution entre la deuxième et la quatrième semaine. Il en est de même pour le lot témoin $\mathrm{T} 0$ entre la huitième et la douzième semaine et pour les animaux du lot $\mathrm{T} 3$ entre la sixième et la huitième semaine. Les animaux soumis au traitement T3 ont obtenu le meilleur GMQ $(117,42 \pm$ $31,04 \mathrm{~g} / \mathrm{jr})$ suivi de T2 $(109,57 \pm 26,98 \mathrm{~g} / \mathrm{jr})$; $\mathrm{du}$ témoin T0 $(102,85 \pm 25,01 \mathrm{~g} / \mathrm{jr})$ et enfin $\mathrm{T} 1(86,21 \pm 19,47 \mathrm{~g} / \mathrm{jr})$.

\section{Rendement carcasse}

Après abattage, habillage et éviscération on constate que, le poids moyen de carcasse du traitement T1 est le plus élevé $(8,60 \pm 0,42 \mathrm{~kg})$, suivi de celui du traitement T3 $(8,34 \pm 1,17 \mathrm{~kg})$, du témoin T0 $(7,96 \pm 1,03$ $\mathrm{kg})$ et enfin T2 $(7,24 \pm 0,34 \mathrm{~kg})$. Cependant la différence n'est pas significative entre le poids des carcasses obtenus avec les différents traitements au seuil de 5\% (P=0,68). Les rendements carcasses moyens des animaux de différents traitements sont présentés par la Figure 2. 
Tableau 2 : Composition chimique des aliments.

\begin{tabular}{|c|c|c|c|c|c|c|c|}
\hline \multirow[t]{2}{*}{ Aliments } & \multirow[t]{2}{*}{ MS (\%) } & \multicolumn{6}{|c|}{ Composition chimique en $\%$ de matière sèche } \\
\hline & & MO & MM & CB & MG & MAT & ENA \\
\hline $\begin{array}{l}\text { Fane de } \\
\text { niébé }\end{array}$ & 95,95 & 89,64 & 6,30 & 35,14 & 1,73 & 17,21 & 35,55 \\
\hline Son de blé & 93,36 & 86,33 & 7,02 & 15,42 & 3,33 & 18,71 & 48,85 \\
\hline
\end{tabular}

MS : Matière sèche ; $\mathrm{MO}$ : Matière organique; MM : Matière minérale ; MG : Matière grasse ; MAT : Matière azotée totale ; $\mathrm{CB}:$ Cellulose brute ; $\%$ ENA : Extractif non azoté = \% MO- (\% CB+\% MAT+\% MG).

Tableau 3 : Consommation alimentaire et indice de consommation.

\begin{tabular}{lllll}
\hline Paramètres & lot T0 & lot T1 & lot T2 & lot T3 \\
\hline Ca fane $(g)$ & $417,30^{\mathrm{a}}$ & $412,60^{\mathrm{a}}$ & $411,30^{\mathrm{a}}$ & $414,00^{\mathrm{a}}$ \\
\hline Ca son $(\mathbf{g})$ & $277,50^{\mathrm{a}}$ & $271,20^{\mathrm{a}}$ & $273,40^{\mathrm{a}}$ & $279,80^{\mathrm{a}}$ \\
\hline IC fane & 9,12 & 8,66 & 8,50 & 8,06 \\
\hline IC son & 6,06 & 5,69 & 5,65 & 5,45
\end{tabular}

Les moyennes marquées par la même lettre (a) sur la même ligne ne sont pas significativement différentes au seuil de 5\% selon le test de Duncan. CA : Consommation Alimentaire ; IC : Indice de Consommation.

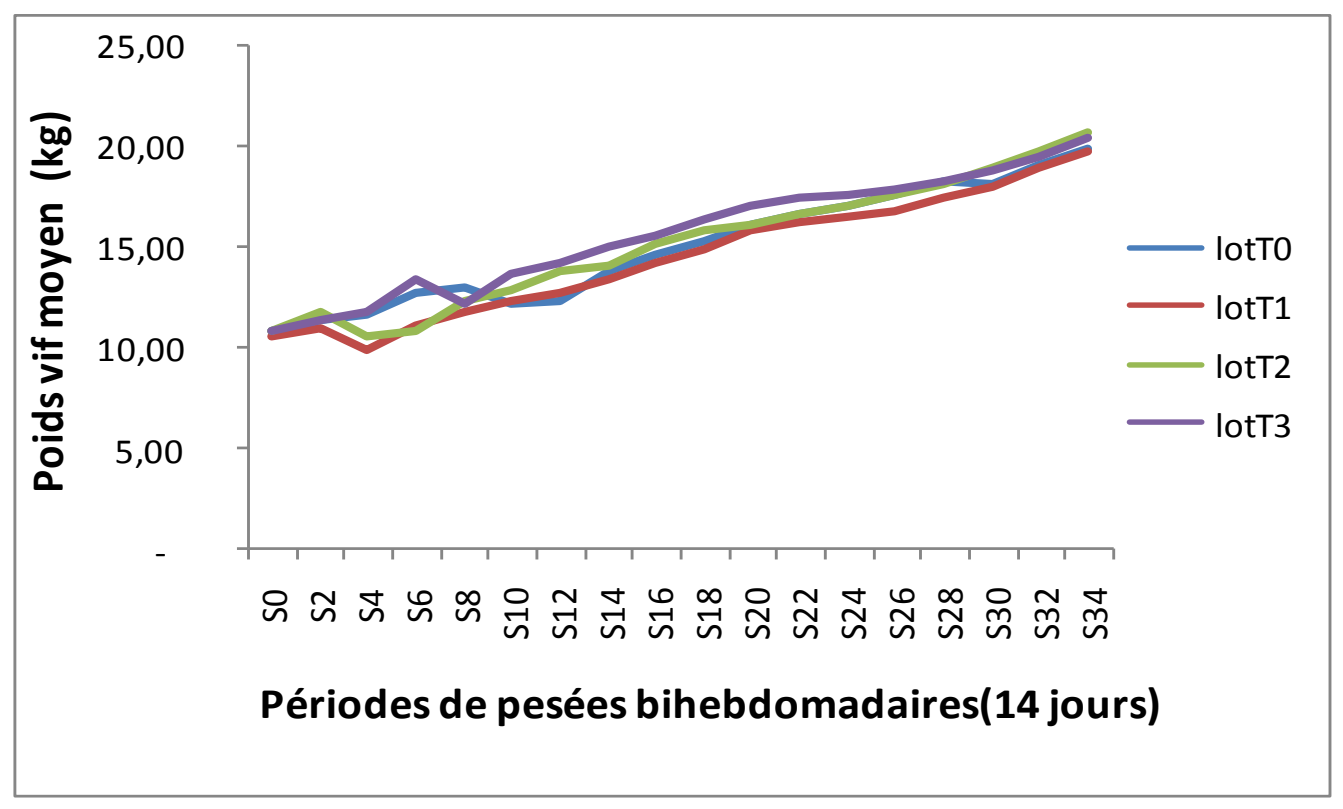

Figure 1 : Evolution du poids vif moyens des différents lots. 


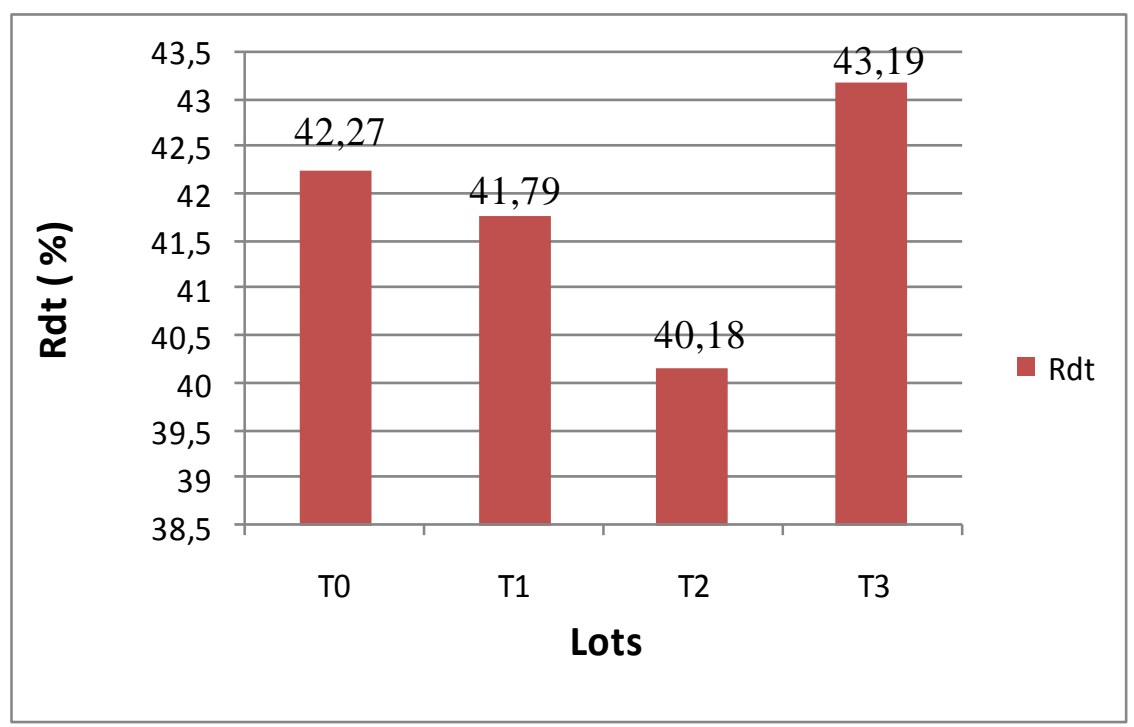

Figure 2 : Rendements carcasses des différents lots.

\section{DISCUSSION}

Le fait de déterminer la composition chimique des ingrédients alimentaires utilisés pour formuler les différentes rations alimentaires permet de connaître la valeur nutritionnelle des rations. Mieux, l'analyse bromatologique des rations alimentaires sert aussi à confirmer ou infirmer les valeurs nutritionnelles obtenues de façon tabulaire. La composition chimique d'un aliment n'a de réel intérêt que si l'aliment est consommé par l'animal à qui, il est servi. Les teneurs en matière sèche (MS), matière azoté totale (MAT) et cellulose brute (CB) obtenus dans cette étude pour les fanes de niébé respectivement $95,95 \% ; 17,21 \%$ et $35,14 \%$ sont plus élevées que celles de Mahaman et al. (2017) qui ont reçus respectivement 91,99\% ; $15,63 \%$ et $33,34 \%$. De même pour le son de blé, $93,36 \%$; $18,71 \%$ et $15,42 \%$ enregistré pour cette étude sont supérieurs à 89,95 ; 19,26 et $14,89 \%$ obtenu par Mahaman et al. (2017). Noblet et Le Goff (2000) et Boudouma (2007) ont obtenu des taux de CB 10 et $10,39 \%$ pour le son de blé, inférieurs aux teneurs déterminés dans la présente étude. Malam Abdou et al. (2017) ont rapporté dans leur étude portant sur l'analyse technicoéconomique des aliments densifiés sur les performances de croissances des boucs roux de Maradi au Niger des taux de $88 \%$ de MS, $33 \%$ de CB et $8,8 \%$ de MAT pour les fanes de niébé. Pour le son de blé il ressort de leur analyse respectivement des teneurs de $91 \%$, $11 \%$ et $16,5 \%$ de MS, CB et MAT. Cette différence de taux peut être expliquée par plusieurs facteurs dont l'âge de la plante, le stade de coupe, la nature des sols des différentes zones d'étude mais aussi aux variétés des cultures. La supplémentation alimentaire en acide malique à 3,6 et $9 \mathrm{~g} / \mathrm{kg}$ incorporés dans le son de blé chez les chevreaux roux n'a significativement modifié ni la croissance pondérale, ni la consommation alimentaire. Ces résultats sont en accord avec ceux d'Aksu et al. (2012) chez qui la supplémentation alimentaire en acide malique à $3 \mathrm{~g} / \mathrm{kg}$ et $6 \mathrm{~g} / \mathrm{kg}$ chez les agneaux sevrés pendant 50 jours n'a pas significativement modifié ni les performances de croissance, ni la consommation alimentaire, ni l'efficacité alimentaire. Carro et al. (2006) n'ont également observé aucun effet de la supplémentation alimentaire en acide malique dans des concentrés chez des agneaux en croissance. L'indice de consommation diminue avec l'addition des doses élevées d'acide malique. Cependant Hani et al. (2018) ont montré qu'un apport de $30 \mathrm{~g}$ d'acide malique par vaches laitières Holstein diminue 
la consommation alimentaire et augmente la production laitière. Les résultats sur l'indice de consommation de son de blé notamment 6,$06 ; 5,69 ; 5,65 ; 5,45$ correspondant respectivement aux lots $\mathrm{T} 0, \mathrm{~T} 1, \mathrm{~T} 2$ et $\mathrm{T} 3$ sont inférieurs à ceux de 12,$76 ; 8,42 ; 7,65$ et 4,17 trouvé par Nouhou (2014) chez la chèvre rousse de Maradi et ceux de Kiema et al. (2008) dans l'étude de la valorisation des ressources alimentaires locales dans l'embouche ovine paysanne, qui sont de 13,$08 ; 10,23 ; 8,99$ et 7,46. Ces différences d'indice peuvent être liées non seulement à la nature et la capacité d'ingestion des aliments distribués mais aussi à la transformation de ces derniers. Les indices de consommation de fane $(9,12)$ et du son de blé $(6,06)$ obtenus chez les animaux témoins sont supérieurs à ceux des animaux dont les rations contiennent des doses d'acide malique. Cette différence d'indice entre le lot témoin et les lots ayant reçu l'acide malique peut être liée au niveau d'ingestion des aliments mais aussi à la présence ou l'absence de l'additif. En effet, les aliments supplémentés en acide malique à $6 \mathrm{~g} / \mathrm{kg}$ et $9 \mathrm{~g} / \mathrm{kg}$ augmentent la consommation alimentaire chez les boucs Roux de Maradi. L'incorporation de la dose $6 \mathrm{~g} / \mathrm{kg}$ d'acide malique donne la meilleure évolution pondérale. Gohier et al. (2017) ont déterminé que l'association d'acide formique et d'acide propionique dans l'eau de boisson pourrait avoir un intérêt en élevage de lapin puisqu'il a permis d'améliorer les performances de croissance. Les GMQ trouvé de l'étude (86,21 à $117,42 \mathrm{~g} / \mathrm{jr}$ ) sont plus intéressant que ceux obtenus par Gnanda et al. (2015) sur des ovins et qui ont oscillé entre 72 à $79 \mathrm{~g} / \mathrm{jr}$. (Zoundi, 2005) dans une étude sur les systèmes d'alimentation des ruminants au Burkina affirme que les jeunes animaux qui sont toujours en phase de croissance, expriment habituellement les meilleurs GMQ surtout lorsqu'ils sont soumis à une alimentation à haute valeur nutritive. Le meilleur GMQ observé au niveau du lot T3 peut s'expliquer par une bonne adaptation à cette ration par les animaux. Les valeurs de rendement carcasse enregistrées dans la présente étude $(40,18$ à 43,19\%) restent globalement plus élevées que celles rapportées par Zoundi et al. (2005) sur des ovins mâles entiers de type Peulh et des ovins Djallonké type Mossi et celles relevées par Nouhou (2014) sur des chèvres rousses. Les résultats obtenus par les premiers auteurs ont varié de $38,20 \%$ à $41,00 \%$ et ceux rapportés par le second ont varié entre 34,58 à $39,56 \%$. En revanche les valeurs obtenues dans cette étude sont inférieurs à ceux enregistrés par Dahouda et al. (2013) qui ont reçus des taux variant de 55,12 à 55,39\%. Ces différences peuvent être liées aux performances pondérales et à l'espèce des animaux.

\section{Conclusion}

Au terme de ce travail qui a évalué l'effet de l'acide malique sur la croissance pondérale et le rendement carcasse des chevreaux Roux, il est apparu, que les animaux soumis au traitement T3 ont obtenu le meilleur gain moyen quotidien GMQ suivi des animaux du lot $\mathrm{T} 2$, puis les animaux du lot témoin $\mathrm{T} 0$ et ceux du lot $\mathrm{T} 1$. L'incorporation dans le son de blé, des doses de $3 ; 6$ et $9 \mathrm{~g} / \mathrm{kg}$ d'acide malique n'a pas significativement affecté les performances de croissance, la prise alimentaire chez les boucs Roux par rapport au témoin. Cette étude a ainsi montré que l'acide malique peut être incorporé chez les boucs roux à une dose allant jusqu'à $9 \mathrm{~g} / \mathrm{kg}$ de concentré en matière sèche dans la ration alimentaire et permettre ainsi l'assimilation des aliments et l'obtention d'une bonne performance de croissance.

\section{CONFLIT D'INTERETS}

Les auteurs déclarent qu'ils n'ont aucun conflit d'intérêts.

\section{CONTRIBUTION DES AUTEURS}

Dans le cadre de cette étude, ABA a participé à l'élaboration du protocole de recherche, la collecte, le traitement des données et la rédaction du manuscrit sous la supervision et l'encadrement du Pr MC. Dr OMMM a contribué fréquemment à la relecture du manuscrit dans le but de l'améliorer et MEN a donné des bonnes propositions et des conseils tout le long des travaux. 


\section{REMERCIEMENTS}

Aux termes de ce travail, nous tenons à remercier le Programme de Productivité Agricole en Afrique de l'Ouest (PPAAO/WAAPP) qui a financé cette étude. Nos remerciements vont également à l'endroit du Directeur du Cendre Secondaire d'Elevage Caprin de Maradi de nous avoir permis de réaliser ces travaux dans leurs locaux.

\section{REFERENCES}

Abdou H. 2014. Complémentation précoce en colostrum de vache Azawak chez la chèvre rousse de Maradi au Niger : effets sur les performances de croissance et de reproduction, et sur la survie au cours de la première année de vie. Thèse pour le grade de Docteur en Sciences Vétérinaires. Université de Liège, Liège, p. 151.

AFSSA. 2007. Propositions pour une démarche d'évaluation de substances ou de produits « nouveaux » destinés à l'alimentation animale: Cas particulier des substances et produits à base de plantes. Maisons-Alfort cedex : Republique Française.

Aksu DE, Sahin T, Kaya I, Unal Y. 2012. Effects of supplementation with different amounts of malic acid to Tuj lambs diets on fattening performance, rumen parameters and digestibility. Revue Méd. Vét., 163 (2): 70-75.

Ali LP, Bossche VD, Thys E. 2003. Enjeux et contraintes de l'élevage urbain et périurbain des petits ruminants à Maradi au Niger : quel avenir? Revue Élev. Méd. Vét. Pays Trop., 56 (1-2) : 73-82.

Boudouma D. 2007. Valeur nutritionnelle du son de blé chez le poulet de chair soumis au stress thermique. Cahiers Agricultures, 16 (6) : 465-468.

Carro, MD, Ranilla MJ. 2003. Influence of different concentrations of disodium fumarate on methane production and fermentation of concentrate feeds by rumen micro-organisms in vitro. $\mathrm{Br} . \mathrm{J}$. Nutr., 90: 617-623.

Carro MD, Ranilla MJ, Giráldez FJ, Mantecón AR. 2006. Effects of malate on diet digestibility, microbial protein synthesis, plasma metabolites, and performance of growing lambs fed a high- concentrate diet. J. Anim. Sci., 84: 405-410.

CIRDES. 2005. Technique de conservation des fourrages par voie humide. Dans Production animale en Afrique de l'Ouest. Fiche $\mathrm{n}^{\circ}$ 18. INERA Burkina Faso : Burkina Faso ; 1 - 4.

Dahouda M, Adjolohoun S, Senou M, Toleba SS, Abdou M, Vidjannagni DS, Kpodekon M, Youssao AKI. 2013. Effets des aliments contenant les feuilles de Moringa oleifera Lam et des aliments commerciaux sur les performances de croissance des lapins (Oryctolagus cuniculus) et la qualité de la viande. Int. J. Biol. Chem. Sci., 7(5): 1838-1852. DOI: http://dx.doi.org/10.4314/ijbcs.v7i5.5.

Gnanda BI, Nignan M, Ouedraogo S, Wereme N'diaye A, Traore O, Sinon B. 2015. Influence d'une co-construction de rationnement amélioré sur les performances d'embouche ovine paysanne dans la commune rurale de Korsimoro au Burkina Faso. Int. J. Biol. Chem. Sci., 9(3) : 1544-1556. DOI : http://dx.doi.org/10.4314/ijbcs.v9i3.35.

Gohier C, Menini FX, Salaün JM, Bourdillon A, Perdriau A, 2017. Effet de l'utilisation d'acides organiques en solution buvable sur les performances de croissance et la mortalité de lapins en engraissement. 17èmes Journées de la Recherche Cunicole, Le Mans : France ; 135-138.

Hani MZ, Ahmed EK, Dyaaeldin AM, Osama HM, Uchenna YA, Sobhy MS, 2018. Enhancing lactational performance of Holstein dairy cows under commercial production: malic acid as an option. $J \mathrm{Sci}$ Food Agric., 99: 885-892.

Houngbo HY, Basso ACPR, Anato Afora F, Sinzogan A, Saidou A, Vayssieres JF, Azokpota P, 2018. Effet de la densité des fourmis rouges [(Oecophyla longinoda latreille (Hymenoptera : formicidae)] des manguiers sur la teneur en sucres et acides organiques de la mangue [Mangifera indica L. (Sapindales: Anacardiaceae)]. Int. J. Biol. Chem. Sci., 
12(6):

2885-2900.

DOI:

https://dx.doi.org/10.4314/ijbcs.v12i6.32.

Indresh HC. 2007. Organic acids, plant extracts. Feed Internat., $9: 10-12$.

Kiema A, Nianogo AJ, Ouédraogo T, Somda J. 2008. Valorisation des ressources alimentaires locales dans l'embouche ovine paysanne: performances technicoéconomiques et options de diffusion. Cahiers Agricultures, 17(1): 23 - 27.

Lila ZA, Mohammed N, Tatsuoka N, Kanda S, Kurokawa Y, Itabashi H. 2004. Effect of cyclodextrin diallyl maleate on methane production, ruminal fermentation and microbes in vitro and in vivo. Anim. Sci. $J ., 75: 15-22$.

Mahaman MMO, Chaibou M, Abdou D, Abdou Ide B ; Issoumane Sitou M ; Mani M. 2017. Evaluation de l'ingestibilité des bouchons alimentaires à base des sous-produits de niébé utilisés pour l'engraissement des ovins. Revue des BioRessources., 7 (2) : 1-11.

Mainet G. 1965. L'élevage dans la région de Maradi (République du Niger). Les Cahiers d'Outre-mer, 18 (69) : 32-72.

Malam Abdou M, Issa S, Dan Gomma A, Sawadogo GJ. 2017. Analyse technicoéconomique des aliments densifiés sur les performances de croissances des boucs roux de Maradi au Niger. Int. J. Biol. Chem. Sci., 11(1) : 280-292. DOI : http://dx.doi.org/10.4314/ijbcs.v11i1.22.

Marc NA. 2001. Contribution à l'évaluation technique du projet à la sélection, la promotion et la diffusion de la chèvre rousse de Maradi-Niger. Thèse de Docteur Vétérinaire, Université Cheikh Anta-Diop de Dakar, Dakar, p. 102.

Moussa S. 2011. Impact de l'élevage de la chèvre rousse de Maradi sur le statut socio-économique de la femme rurale au Niger. Mémoire de Master II, Université Cheikh Anta-Diop de Dakar, Dakar, p. 30.

Newbold, CJ, Lopez S, Nelson N, Ouda JO, Wallace RJ, Moss AR. 2005. Propionate precursors and other metabolic intermediates as possible alternative electron acceptors tomethanogenesis in ruminal fermentation in vitro. $B r . J$. Nutr., 94 : 27-35.

Noblet J, Le Goff G. 2000. Utilisation digestive et valeurs énergétiques du blé, du maïs et leurs co-produits chez le porc en croissance et la truie adulte. Journées Rech. Porcine en France, 32 : 177-183.

Nouhou ME. 2014. Effets d'une substitution du tourteau de graines de coton par les gousses de Piliostigmareticulatum (De.Candolle) Hochstetter dans l'alimentation, sur les performances de croissance de la chèvre rousse de Maradi. . Mémoire de Master. Ecole Inter-Etats des Sciences et Médecine Vétérinaires De Dakar, Dakar, p. 30.

OCHA. 2014. Profil humanitaire régional de Maradi. Bureau des Nations Unies de la Coordination des Affaires Humanitaires : NIGER ; 1-4.

Papatsiros VG, Christodoulopoulos G. 2011. The use of organic acids in rabbit farming. Online J. Anim. Feed Res., 1(6): 434-438.

Wallace RJ, Wood TA, Rowe A, Price J, Yanez DR, Williams SP, Newbold CJ. 2006. Encapsulated fumaric acid as a means of decreasing ruminal methane emissions. Int. Congr. Ser., 1293 :148151.

Zoundi JS, Sawadogo L, Nianogo AJ. 2005. Utilisation de blocs multinutritionnels en substitution partielle de concentre pour l'engraissement des ovins au sein des systèmes mixtes agriculture-élevage du plateau central du Burkina Faso. J. Sci., 5(1) : 17-22.

Zoundi JS. 2005. Systèmes d'Alimentation des ruminants au sein des exploitations mixtes agriculture élevage du plateau central au Burkina-Faso. Thèse de doctorat d'Etat, Université de Ouagadougou, Ouagadougou, p.179. 